\title{
Evaluation of pentraxin 3 level and cardiac functions in psoriatic children
}

\author{
Mecnun Çetin ${ }^{1 \oplus}$, İbrahim Halil Yavuz², Göknur Özaydın Yavuz², \\ Kamuran Karaman ${ }^{3}$ \\ Departments of ${ }^{1}$ Pediatric Cardiology, ${ }^{2}$ Dermatology and ${ }^{3}$ Pediatric Hematology, Van Yüzüncü Yıl University Faculty of Medicine, \\ Van, Turkey.
}

\begin{abstract}
Psoriasis is a chronic inflammatory disorder affecting the skin, nails, and joints. Its lifetime prevelance has been estimated to be at $1 \%$ to $3 \%$. This study was designed to examine the association between serum pentraxin 3 (PTX3) and cardiovascular function in psoriatic children. Thirty-three children who were diagnosed with psoriasis, and 29 healthy children, between 4 and 18 years of age, were included in the study. Both patient and control group was evaluated by the pulsed wave tissue doppler imaging (TDI) echocardiography as well as with conventional Doppler echocardiography (CDE). PTX3 values of the groups were evaluated. There was no difference between cases and controls for age $(9.67 \pm 3.72,9.60 \pm 2.84$ years, $\mathrm{p}=0.916$, respectively). In evaluation of the left ventricle (LV) CDE; A wave, isovolumic relaxation time (IVRT) and myocardial performance index (MPI) were significantly higher in the study group ( $<<0.05)$. Ejection time (ET) was significantly lower in the study group compared to the control group ( $\mathrm{p}<0.05$ ). In evaluation of LV TDI; Deceleration time ( $\left.\mathrm{DT}^{\prime}\right), \mathrm{IVRT}^{\prime}$, $\mathrm{E} / \mathrm{E}^{\prime}$ and MPI' were found to be significantly higher in the study group (p<0.05). In addition to, $\mathrm{E}^{\prime}, \mathrm{E}^{\prime} / \mathrm{A}^{\prime}$ and $\mathrm{ET}^{\prime}$ were siginificantly lower in study group. PTX3 level was significantly higher in the study group compared to the control group ( $\mathrm{p}=0.009$ ) (Table III). However, no correlation was found between PTX3 level and cardiovascular parameters. In conclusion; both doppler echocardiography and PTX3 may be useful tools for the screening of cardiovascular (CV) risk in these patients. Psoriasis itself may be an independent risk factor for cardiac dysfunction in the pediatric population.
\end{abstract}

Key words: tissue doppler echocardiography, pediatric, psoriasis, pentraxin 3.

Psoriasis is a chronic inflammatory disease affecting the skin, nails, and joints. Its lifetime prevelance has been estimated to be at $1 \%$ to $3 \%{ }^{1}$ Currently, psoriasis is accepted as a systemic inflammatory disorder associated with various medical comorbidities. ${ }^{2}$ Several epidemiological investigations have reported that adult patients with psoriasis have elevated prevalence of wellknown cardio-metabolic risk factors such as diabetes, hypertension, dyslipidemia, obesity and metabolic syndrome compared with the general population, and importantly a clinically marked increased risk of cardiovascular (CV) disease and cardiovascular mortality. ${ }^{3-5}$

$凶$ Mecnun Çetin

drmecnun@hotmail.com

Received 17th September 2018; revised 5th Januray 2019; accepted 18th February 2019
Data related with cardiovascular risk in childhood psoriasis is limited, however there are many studies that shows the childhood psoriasis is also associated with an increased occurrence of cardiovascular comorbidities. ${ }^{6}$

PTX3 is an inflammatory molecule and a member of pentraxin superfamily which contains C-Reactive Protein (CRP) and serum amyloid P.7 PTX3 is produced by different kinds of cells, mainly by dendritic cells, macrophages, fibroblasts, activated endothelia, and by other tissues. It is synthesized in response to proinflammatory stimulants, including bacteria, IL-1, and TNF-alpha produced by primarily endothelial cells, neutrophils, and macrophages. ${ }^{8}$ PTX3 has similarity with regards to the structure and actions of CRP which is known as an acute phase reactant. Higher 
PTX3 levels were detected during infections, autoimmune diseases, inflamatory conditions and various vasculitis. ${ }^{9}$ Serum level of PTX3 especially in children with psoriasis has not yet been investigated.

The association between psoriasis and cardiovascular disease may be explained by sharing chronic systemic inflammatory processes. Investigations with psoriasiform experimental models have showed that sustained skin-specific inflammation is related with increased aortic root vascular inflammation and arterial thrombosis. ${ }^{10}$ The present study was designed to examine the association between serum PTX3 and cardiovascular status in psoriatic children.

\section{Material and Methods}

Thirty tree children with psoriasis and 29 age and sex matched controls were recruited from Pediatric Cardiology and Dermatology Clinics. Patients with a murmur in the pediatric cardiology outpatient clinic that had no other illnesses were accepted in the control group.

Psoriasis was diagnosed by history, clinical examination and histopathological findings. Psoriasis Area Severity Index (PASI) scoring was performed for the severity of the disease. The patients accepted to the study were those who had not received any treatment for the last month. Height, weight, body mass index (BMI), heart rate and blood pressure were measured in both groups.

Both patient and control groups were evaluated using the pulsed wave tissue doppler echocardiography, as well as with conventional doppler echocardiography (CDE). The PTX3 values of both the patient and control group were evaluated.

Patients with any cardiac disease, systemic inflammatory disease, infectious disease, obesity, or immunological disease were excluded from the study.
All patients' parents gave informed consent to be involved in this study after the study protocol was explained to them before the initiation of the study.

\section{Echocardiography}

Echocardiography was performed in the left lateral decubitus and supine position with an ultrasound machine GE Vivid 6S system (GEVingmed Ultrasound AS, Horten, Norway) and 3S-RS (3.5 MHz) probe. Averages of three consecutive cycles for all echocardiographic data were measured. Images were obtained from parasternal and apical views using 2D, M-mode and doppler echocardiographic techniques. Examinations were performed by a single pediatric cardiologist. M-mode measurements were evaluated according to standards outlined by the American Society of Echocardiography. ${ }^{11}$ Interventricular septum diameter (IVSD) and left ventricular posterior wall diameter (LVpWD) was measured at the end of diastole in parasternal long axis. Left ventricular end-diastolic and end-systolic diameter was measured at the end of systole and diastole in parasternal long axis. Left ventricular (LV) global systolic function was assessed as the mitral annular plane systolic excursion (MAPSE) by the 2-dimensional difference of the end- diastolic and end-systolic lines traced between the center of the ultrasound fan origin and the junction of the LV lateral mitral annulus in the apical $4 \mathrm{C}$ view

Conventional doppler echocardiography and Tissue Doppler imaging (TDI) values for all patients were recorded.

Conventional Doppler echocardiography examination: The cursor was placed in the parasternal long-axis view at the junction of the LV exit pathway with the anterior mitral valve to obtain mitral flow and LV outflow pathway simultaneously. E-velocity, A-velocity and deceleration time (DT), isovolumic relaxation time (IVRT) were measured from diastolic function parameters. E velocity; The peak velocity of the fast filling phase of $\mathrm{LV}, \mathrm{A}$ 
velocities; was taken as the peak velocity of late ventricular filling flow and the E/A ratio was calculated. DT was measured as the time between peak $\mathrm{E}$ velocities and the point at which the fast filling ends. S-wave, ejection time (ET) and isovolumic contraction time (IVCT) of systolic function parameters were measured.

TDI was recorded from the apical four-chamber view with the pulse wave Doppler sample volume placed on the mitral lateral annulus. Peak systolic $\left(S^{\prime}\right)$ velocity, peak early $\left(E^{\prime}\right)$, the deceleration time of $\mathrm{E}^{\prime}$ wave $\left(\mathrm{DT}^{\prime}\right)$ and peak late diastolic myocardial annular velocity $\left(\mathrm{A}^{\prime}\right)$, isovolumic relaxation time (IVRT'), and isovolumic contraction time (IVCT') were measured. Myocardial performance index (MPI') of LV was calculated with the Tei index Formula. ${ }^{12}$ (Fig. 1).

\section{Pentraxin 3 (PTX3)}

Samples for PTX3 were taken into anticoagulantfree biochemical tubes, the samples were centrifuged at $1500 \mathrm{rpm}$ for 10 minutes after the coagulation process was completed, separated into their sera and stored at $-40 \mathrm{C}$ until the day of assesment. The levels of PTX3 was measured with the immunoabsorbent method bound to double antibody enzyme sandwich model in ELISA (Eastbiopharm ${ }^{\circledR}$, Zhejiang, China). The results were measured as $\mathrm{ng} / \mathrm{ml}$ for PTX3.

Ethics approval was obtained from the Regional Ethics Board with the decision number 98 of 04.08.2017.

\section{Statistical analysis}

The studied variables (characteristics) were presented as mean, minimum and maximum values. Student $\mathrm{t}$ test was used to compare Control and Patient group means for the studied variables. Pearson correlation analysis was carried out to examine linear relationships among the variables were considered as $5 \%$ and SPSS (ver: 21) statistical program was used for all statistical computations.

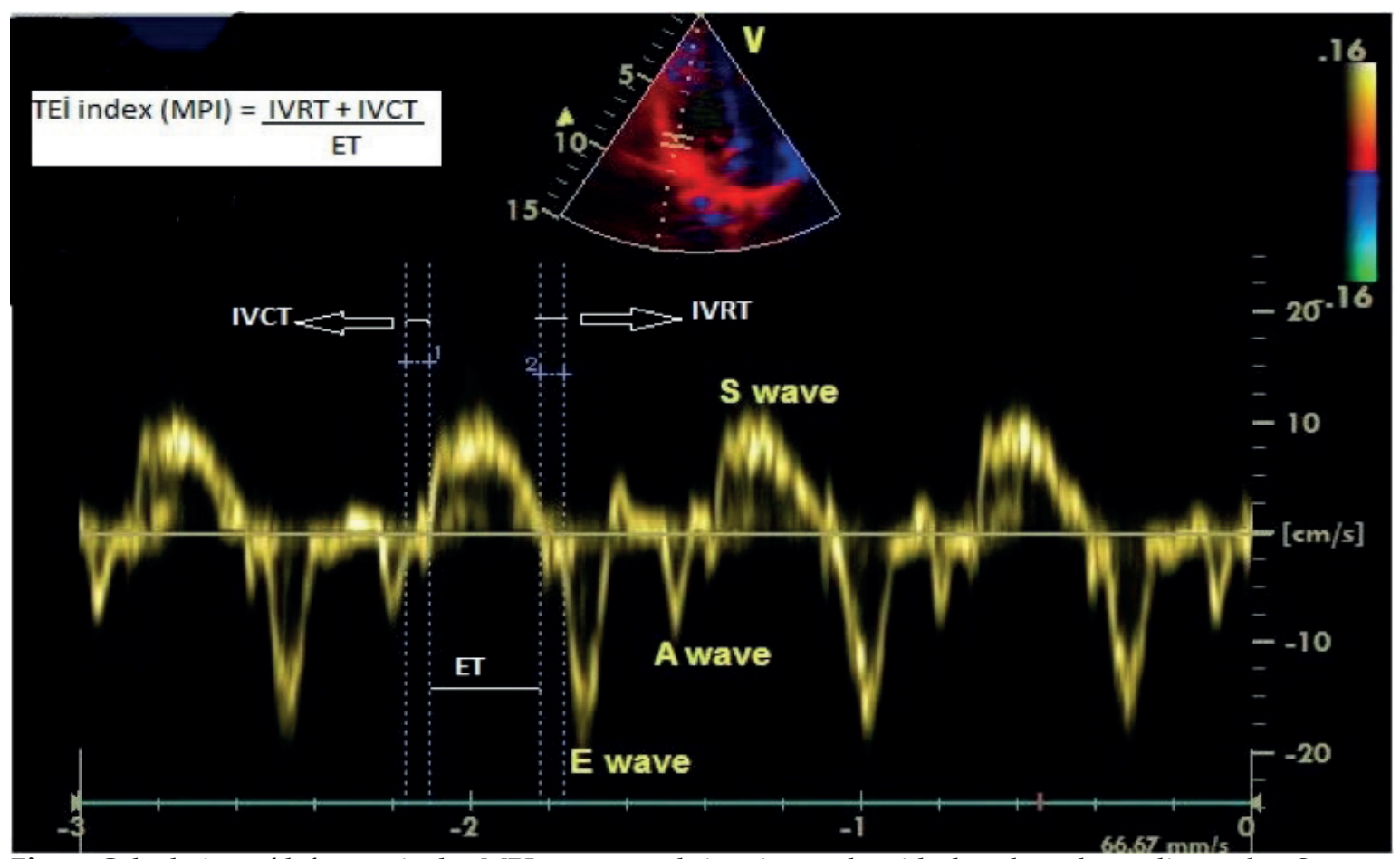

Fig. 1. Calculation of left ventricular MPI, waves and time intervals with doppler echocardiography. S wave, systolic myocardial velocity; E wave, early diastolic myocardial velocity; A wave, late diastolic myocardial velocity; IVRT, isovolumic relaxation time; IVCT, isovolumic contraction time; ET, ejection time; MPI, myocardial performance index. 


\section{Results}

This study included 33 psoriatic children and 29 healthy controls. There was no difference between cases and controls for age $(9.67 \pm 3.72$, $9.60 \pm 2.84$ years, $p=0.916$, respectively). Mean psoriasis duration time was $2.6 \pm 1.9$ years. Systolic blood pressure (sBP) was significantly higher in study group. Comparison between weight, BMI, height, $\mathrm{dBP}$ and heart rate revealed no statistically significant difference betwen groups (Table I).

\section{Echocardiographic data}

Comparison between LVedD, LVesD, LVpWD, IVSD and MAPSE revealed no statistically significant difference between groups in M-mode echocardiographic examination $(\mathrm{p}>0.05)$ (Table I).

In evaluation of LV convantional doppler echocardiography; A wave, IVRT and MPI were significantly higher in study group ( $p$ $<0.05)$. ET was significantly lower in study group compared to control group ( $p<0.05)$. No significant difference were detected between the study group and control group regarding all other variables $(\mathrm{p}>0.05)$ (Table II).

In evaluation of LV TDI; DT', IVRT', E/E' and MPI' were found to be significantly higher in the study group $(p<0.05)$. In addition, $E^{\prime}, E^{\prime} / A^{\prime}$ and $\mathrm{ET}^{\prime}$ were siginificantly lower in the study group (Table III).

PTX3 level was significantly higher in the study group compared to the control group $(p=0.009)$ (Table III). Although the PTX3 level was higher in the study group, no significant correlation was found between PTX3 and MPI, MPI', PASI, disease duration, MAPSE and other echocardiographic parameters (Table IV).

\section{Discussion}

This study was designed to examine the association between serum PTX3 and cardiovascular condition in psoriatic children. Our study is the first study which measure PTX3 level and examine cardiac functions with TDI in psoriatic children. In our study, we documented the occurrence of subclinical systolic and diastolic deterioration in psoriatic children.

Table I. General characteristics and m-mode echocardiographic findings of groups.

\begin{tabular}{lccc}
\hline & Study $(\mathrm{n}=33)$ & Control $(\mathrm{n}=29)$ & $\mathrm{P}$ \\
\hline Age $($ years $)$ & $9.69 \pm 3.72$ & $9.60 \pm 2.84$ & 0.916 \\
Sex $(\mathrm{M} / \mathrm{F})$ & $17 / 16$ & $14 / 15$ & \\
Weight $(\mathrm{kg})$ & $33.39 \pm 16.33$ & $32.38 \pm 14.23$ & 0.557 \\
Height $(\mathrm{cm})$ & $132.5 \pm 20.5$ & $135.0 \pm 17.3$ & 0.615 \\
BMI $\left(\mathrm{kg} / \mathrm{m}^{2}\right)$ & $17.8 \pm 3.9$ & $16.6 \pm 2.6$ & 0.151 \\
Psoriasis duration $(\mathrm{y})$ & $2.6 \pm 1.9$ & & \\
PASI & $5.9 \pm 4.2$ & & \\
sBP $(\mathrm{mmHg})$ & $109.0 \pm 10.2$ & $102.2 \pm 7.9$ & 0.006 \\
dBP $(\mathrm{mmHg})$ & $67.6 \pm 11.4$ & $65.5 \pm 8.3$ & 0.401 \\
HR $($ beat $/ \mathrm{min})$ & $88.5 \pm 19.7$ & $84.8 \pm 12.4$ & 0.398 \\
LVedD $(\mathrm{mm})$ & $38.3 \pm 9.1$ & $36.6 \pm 3.8$ & 0.352 \\
LVesD $(\mathrm{mm})$ & $23.7 \pm 4.5$ & $22.8 \pm 2.9$ & 0.372 \\
LVpW $(\mathrm{mm})$ & $4.4 \pm 1.0$ & $4.0 \pm 0.7$ & 0.069 \\
IVSD $(\mathrm{mm})$ & $4.2 \pm 1.0$ & $3.9 \pm 0.7$ & 0.125 \\
MAPSE $(\mathrm{mm})$ & $12.9 \pm 1.8$ & $13.0 \pm 1.7$ & 0.745 \\
\hline
\end{tabular}

BMI: Body mass index; sBP: sistolic blood pressure; dBP: diastolic blood pressure; HR: heart rate; LVedD: left ventricle enddiastolik diameter; LVesD: left ventricle end-sistolik diameter; LVpWD: left ventricle posterior wall diameter; IVSD: interventricular septum diamater; MAPSE: mitral annular plane systolic excursion. 
Table II. Conventional doppler echocardiographic parameters of groups.

\begin{tabular}{lccc}
\hline Parameter & Study $(\mathrm{n}=33)$ & Control $(\mathrm{n}=29)$ & $\mathrm{P}$ \\
\hline $\mathrm{E}(\mathrm{cm} / \mathrm{s})$ & $82.1 \pm 11.6$ & $80.4 \pm 11.7$ & 0.569 \\
$\mathrm{~A}(\mathrm{~cm} / \mathrm{s})$ & $49.9 \pm 13.1$ & $43.2 \pm 9.5$ & 0.026 \\
$\mathrm{E} / \mathrm{A}$ & $1.73 \pm 0.46$ & $1.93 \pm 0.45$ & 0.095 \\
$\mathrm{~S}(\mathrm{~cm} / \mathrm{s})$ & $79.6 \pm 16.26$ & $85.3 \pm 10.8$ & 0.117 \\
DT $(\mathrm{ms})$ & $89.7 \pm 19.5$ & $84.5 \pm 12.9$ & 0.234 \\
ET $(\mathrm{ms})$ & $241.1 \pm 19.4$ & $257.5 \pm 17.0$ & 0.001 \\
IVRT $(\mathrm{ms})$ & $71.2 \pm 16.5$ & $58.9 \pm 12.4$ & 0.002 \\
IVCT $(\mathrm{ms})$ & $66.9 \pm 16.4$ & $68.0 \pm 13.0$ & 0.768 \\
MPI & $0.57 \pm 0.10$ & $0.49 \pm 0.09$ & 0.002 \\
\hline
\end{tabular}

E: early diastolic myocardial velocity; A: late diastolic myocardial velocity; S: systolic myocardial velocity; DT: deceleration time; IVRT: isovolumic relaxation time; IVCT: isovolumic contraction time; ET: ejection time; MPI: myocardial performance index.

Table III. Tissue doppler imaging parameters and PTX3 values of groups.

\begin{tabular}{lccc}
\hline Parameter & Study $(\mathrm{n}=33)$ & Control $(\mathrm{n}=29)$ & $\mathrm{P}$ \\
\hline $\mathrm{E}^{\prime}(\mathrm{cm} / \mathrm{s})$ & $15.33 \pm 3.41$ & $17.55 \pm 2.64$ & 0.006 \\
$\mathrm{~A}^{\prime}(\mathrm{cm} / \mathrm{s})$ & $7.21 \pm 1.89$ & $6.72 \pm 1.19$ & 0.239 \\
$\mathrm{E}^{\prime} / \mathrm{A}^{\prime}$ & $2.21 \pm 0.62$ & $2.67 \pm 0.52$ & 0.003 \\
$\mathrm{E}^{\prime} \mathrm{E}^{\prime}$ & $5.71 \pm 2.21$ & $4.67 \pm 0.94$ & 0.023 \\
$\mathrm{~S}^{\prime}(\mathrm{cm} / \mathrm{s})$ & $9.81 \pm 2.44$ & $9.06 \pm 1.22$ & 0.141 \\
$\mathrm{DT}^{\prime}(\mathrm{ms})$ & $74.90 \pm 12.59$ & $64.03 \pm 11.62$ & 0.001 \\
$\mathrm{ET}^{\prime}(\mathrm{ms})$ & $256.5 \pm 29.3$ & $271.7 \pm 20.8$ & 0.024 \\
$\mathrm{IVRT}^{\prime}(\mathrm{ms})$ & $60.30 \pm 10.36$ & $52.65 \pm 11.19$ & 0.007 \\
IVCT' $^{\prime}(\mathrm{ms})$ & $66.51 \pm 15.33$ & $61.89 \pm 9.23$ & 0.163 \\
MPI' & $0.50 \pm 0.11$ & $0.42 \pm 0.07$ & 0.003 \\
PTX3 $(\mathrm{ng} / \mathrm{ml})$ & $5.89 \pm 5.00$ & $3.10 \pm 2.61$ & 0.009 \\
\hline
\end{tabular}

E': early diastolic myocardial velocity; $\mathrm{A}^{\prime}$ : late diastolic myocardial velocity; $\mathrm{S}^{\prime}$ : systolic myocardial velocity; $\mathrm{DT}^{\prime}$ : deceleration time; IVRT': isovolumic relaxation time; IVCT': isovolumic contraction time; ET': ejection time; MPI': myocardial performance index; PTX3: pentraxin 3.

Table IV. Correlation between parameters.

\begin{tabular}{|c|c|c|c|c|c|c|c|c|c|c|}
\hline & & MPI & $\mathrm{MPI}^{\prime}$ & PASI & Duration & MAPSE & IVRT' & IVRT & $\mathrm{E} / \mathrm{A}$ & $\mathrm{E}^{\prime} / \mathrm{A}^{\prime}$ \\
\hline \multirow[t]{2}{*}{$\overline{\text { PTX3 }}$} & $\mathrm{r}$ & +.130 & +.008 & +.056 & -.073 & +.071 & -.014 & +.169 & +.167 & +.226 \\
\hline & $\mathrm{p}$ & 0.471 & 0.965 & 0.759 & 0.686 & 0.693 & 0.940 & 0.348 & 0.353 & 0.205 \\
\hline
\end{tabular}

E: early diastolic myocardial velocity; A: late diastolic myocardial velocity; IVRT: isovolumic relaxation time; MPI: myocardial performance index; PTX3: pentraxin 3; PASI: Psoriasis area severity index; MAPSE: mitral annular plane systolic excursion.

In recent decades, the association of psoriasis with several comorbidities, such as obesity, metabolic syndrome, systemic hypertension, dyslipidemia, type 2 diabetes, malignancies and inflammatory bowel diseases have been documanted. This association between psoriasis and comorbidities, especially cardiovascular and metabolic diseases, may be related to their chronic and inflammatory nature, especially due to elevated pro-inflammatory cytokines that are mandatory for the occurrence the pathophysiology of such disorders. ${ }^{10,13,14}$ 
Psoriasis is accepted as a systemic inflammatory disease, several studies have evaluated PTX3 and CRP levels in psoriatic patients. Numerous studies have shown that plasma PTX3 levels are increased in patients with psoriasis. ${ }^{7,15,16}$

In our study, PTX3 was significantly increased in psoriatic patients (pointing to possible role in pathogenesis of psoriasis). However, there was no significant correlation between PTX3 and risk factors of psoriasis such as PASI and psoriasis duration. There was also no significant correlation between PTX3 and doppler parameters (Table IV). The finding of no correllation between PTX3 and PASI found in our study may be due to the occurence of systemic inflammation earlier than phenotypic presentations in psoriatic children.

The results of our study showed that echocardiographic parameters were negatively affected in the study group compared to those in the control group. Conventional doppler echocardiography and TDI are important techniques to evaluate cardiac function. Prolongation of IVRT and DT, decrease in maximum $\mathrm{E}$ wave velocity and E/A ratio, and elevation in maximum $\mathrm{A}$ wave velocity show diastolic dysfunction. ${ }^{17}$ Prolongation of IVCT and shortening of ET showed systolic dysfunction. MPI, which is a indicator for both systolic and diastolic functions of the heart, is a parameter which is easy-to-apply, reproducible parameter independent of ventricular geometry, blood pressure, and heart rate changes. ${ }^{18}$ An abnormal increase in MPI, measured by CDE and TDI, indicates ventricular global dysfunction (both systolic and diastolic).

To the best of our knowledge, there are no studies evaluating psoriatic childhood heart with tissue doppler echo. There is only one study done by Salam et al. ${ }^{10}$ with conventional doppler echocardiography. Salam et al. ${ }^{10}$ found that IVRT and DT values were significantly higher in children with psoriasis than the control group. They reported that diastolic dysfunction developed in psoriatic children. In a study by Ozden et al. ${ }^{19}$ which evaluated the adult psoriatic patients with doppler echocardiography stated that there is significant changes in parameters that indicate subclinical cardiac dysfunctions (High MPI, low E/A, and $\mathrm{E} / \mathrm{E}$ ratio). In our study, parameters indicating diastolic dysfunction; $\mathrm{E}^{\prime}$ wave and $\mathrm{E}^{\prime} / \mathrm{A}^{\prime}$ ratio were lower in the study group, DT', IVRT' and $\mathrm{A}$ wave were higher in the study group. ET, the indicator of systolic dysfunction, was significantly lower in the study group. In our study, MPI values measured with both TDI and CDE were significantly higher in study group than the control group (Table II, III). Thus, our result in psoriatic patients shows the systolic and especially diastolic dysfunction of the heart.

Moreover, we can say that MPI which is practically easy to perform can be used as an early marker of cardiac dysfunction in the follow-up of these patients.

In this study, a relatively new and more sensitive method, TDI, was used to detect subclinical cardiac involvement in psoriatic childhood patients. Both doppler echocardiography and PTX3 may be useful tools for the screening of $\mathrm{CV}$ risk in these patients. However, there was no significant relationship between TDI and PTX3 levels. This can be explained by the population size of the study. In these patients, psoriasis may have an independent $\mathrm{CV}$ risk factor, causing cardiac disfunction. In addition, larger and longer-term studies are necessary to evaluate clinical implications of our finding.

\section{REFERENCES}

1. Tollefson MM, Crowson CS, McEvoy MT, Maradit Kremers $\mathrm{H}$. Incidence of psoriasis in children: a population-based study. J Am Acad Dermatol 2010; 62: 979-987.

2. Reich K. The concept of psoriasis as a systemic inflammation: implications for disease management. J Eur Acad Dermatol Venereol 2012; 26(Suppl 2): 3-11.

3. Neimann AL, Shin DB, Wang X, Margolis DJ, Troxel AB, Gelfand JM. Prevalence of cardiovascular risk factors in patients with psoriasis. J Am Acad Dermatol 2006; 55: 829-835. 
4. Armstrong AW, Harskamp CT, Armstrong EJ. Psoriasis and metabolic syndrome: a systematic review and meta-analysis of observational studies. J Am Acad Dermatol 2013; 68: 654-662.

5. Armstrong EJ, Harskamp CT, Armstrong AW. Psoriasis and major adverse cardiovascular events: a systematic review and meta-analysis of observational studies. J Am Heart Assoc 2013; 2: e000062.

6. Torres T, Machado S, Mendonça D, Selores M. Cardiovascular comorbidities in childhood psoriasis. Eur J Dermatol 2014; 24: 229-235.

7. Uysal S, Yilmaz FM, Karatoprak K, Artüz F, Cumbul NU. The levels of serum pentraxin3, CRP, fetuin-A, and insulin in patients with psoriasis. Eur Rev Med Pharmacol Sci 2014; 18: 3453-3458.

8. Inoue K, Kodama T, Daida H. Pentraxin 3: a novel biomarker for inflammatory cardiovascular disease. Int J Vasc Med 2012; 2012: 657025.

9. Bevelacqua V, Libra M, Mazzarino MC, et al. Long pentraxin 3: a marker of inflammation in untreated psoriatic patients. Int J Mol Med 2006; 18: 415-423.

10. Salam MA, Mahran Z, Emran T, Khalik HA. Evaluation of myeloperoxidase level and cardiovascular problems in psoriatic children in Damietta governorate. Res J Cardiol 2015; 8: 12-19.

11. Schiller NB, Shah PM, Crawford $M$, et al. Recommendations for quantitation of the left ventricle by two-dimensional echocardiography. American Society of Echocardiography Committee on Standards, Subcommittee on Quantitation of Two-Dimensional Echocardiograms. J Am Soc Echocardiogr 1989; 2: 358-367.
12. Çetin M, Bozan N. The effects of adenotonsillar hypertrophy corrective surgery on left ventricular functions and pulmonary artery pressure in children. Int J Pediatr Otorhinolaryngol 2017; 101: 41-46.

13. Prey S, Paul C, Bronsard V, et al. Cardiovascular risk factors in patients with plaque psoriasis: a systematic review of epidemiological studies. J Eur Acad Dermatol Venereol 2010; 24(Suppl 2): 23-30.

14. Prey S, Paul C, Bronsard V, et al. Assessment of risk of psoriatic arthritis in patients with plaque psoriasis: a systematic review of the literature. J Eur Acad Dermatol Venereol 2010; 24(Suppl 2): 31-35.

15. Ctirad A, Lenka B, David P, et al. Goeckerman's therapy for psoriasis with special reference to serum pentraxin 3 level. Int J Dermatol 2008; 47: 1011-1014.

16. Okan G, Baki AM, Yorulmaz E, Doğru-Abbasoğlu S, Vural P. Serum visfatin, fetuin-A, and pentraxin 3 levels in patients with psoriasis and their relation to disease severity. J Clin Lab Anal 2016; 30: 284-289.

17. Isaaz K, Thompson A, Ethevenot G, Cloez JL, Brembilla B, Pernot C. Doppler echocardiographic measurement of low velocity motion of the left ventricular posterior wall. Am J Cardiol 1989; 64: 66-75.

18. Tekten T, Onbasılı AO, Ceyhan C, Unal S, Discigil B. Novel approach to measure myocardial performance index: pulsed-wave tissue Doppler echocardiography. Echocardiography 2003; 20: 503510.

19. Ozden HK, Polat M, Ozturk S, Bugdayci G. Assessment of subclinical cardiac damage in chronic plaque psoriasis patients: a case control study. Arch Med Sci Atheroscler Dis 2016; 1: e126-e132. 